\title{
Global Climate Policy and Collective Action: A
}

\section{Comment}

\author{
Amanda Kennard * $\quad$ Keith E. Schnakenberg ${ }^{\dagger}$
}

April 18, 2022

\begin{abstract}
In a recent issue of Global Environmental Politics, Aklin and Mildenberger (2020) argue against the prevailing characterization of climate change cooperation as a problem of free riding or collective action. The authors argue that models of collective action imply (1) policy reciprocity and (2) inaction in the absence of formal agreements to limit free riding. They argue that neither empirical implication is supported by an review of states' climate policy to date. In this comment we note that standard collective action models imply neither of the above hypotheses. As a result the empirical tests advanced in the original article are uninformative as to the explanatory power of the collective action model for international climate politics.
\end{abstract}

\footnotetext{
*Assistant Professor, Department of Political Science, Stanford University. Contact: amanda.kennard@stanford.edu

${ }^{\dagger}$ Assistant Professor of Political Science, Washington University in St. Louis. Contact: keith.schnakenberg@gmail.com.
} 
There exists widespread consensus within the scientific community regarding humankind's responsibility for global climate change as well as the potential for widespread devastation if warming is left unchecked. Still, policy makers have made little progress in slowing worldwide emissions of greenhouse gasses. How do we explain this failure to act? One common answer highlights the role of incentives: climate change mitigation requires costly actions from everyone and the benefits are experienced by everyone regardless of whether they helped achieve it. Since the cost-benefit calculations of national policymakers, firms, and households do not fully internalize the effects of their actions on the welfare of everyone else, the collection of individual actions to mitigate climate change are less than what would be ideal from a global standpoint. This is the global collective action problem.

In a recent issue of Global Environmental Politics, Aklin and Mildenberger (2020) argue that international climate change policy is not characterized by free riding or collective action concerns. Their argument rests on two main claims. First, according to the authors, collective action theories imply an absence of policy action where international institutions are not available to manage free riding. Empirically though the authors argue that countries often take action at the national level despite the absence of strong international institutions. Second, the authors claim that theories of collective action imply reciprocity in the implementation of policies to mitigate climate change, especially as it concerns potential defection by "pivotal" actors. Again, in the authors' view the empirical record contradicts this claim as national publics and governments alike behave in "a largely unconditional fashion" (Aklin and Mildenberger, 2020, p.16).

The stakes of this debate are significant for scholars and practioners. If we accept the falsification of collective action as a framework for understanding climate change mitigation then policy prescriptions primarily designed to reduce free riding would be misguided. Instead efforts to curb carbon emissions via policy should be directed towards the national or sub-national levels. To the contrary, in this note we argue that standard models of collective action do not imply either of the hypotheses advanced in Aklin and Mildenberger (2020). As a result, the empirical findings cannot speak to whether or not models of collective action are effective tools for analyzing the challenges 
of climate change mitigation.

The purpose of this note is primarily methodological. We do not take a position on the relative merits of studying global environmental problems through a collective action versus distributive politics lens. Instead, our criticism of Aklin and Mildenberger (2020) is focused on an issue fundamental to empirical social science: the derivation of predictions from theoretical models. While our substantive focus in this article is on the implications of collective action theory, we feel this is a valuable example for the broader literature of the importance of careful engagement with theoretical models in empirical work.

\section{Aklin and Mildenberger's (2020) Argument}

We begin by reviewing the key elements of Aklin and Mildenberger (2020). The argument proceeds in two steps. First, the authors identify two versions of the collective action model. Adherents of the "strong" model argue that collective action is the fundamental organizing principle of climate (in)action. Proponents of the "weaker" version view collective action as one of many models which may shape states' and policy makers' actions in tandem with other forces. Next, Aklin and Mildenberger (2020) identify three implications of the strong version (SCA1-SCA3) and three implications of the weak version (WCA1-WCA2) which they subsequently use to test the utility of the collective action model.

Throughout Aklin and Mildenberger (2020) also contrast theories of collective action with theories of climate change mitigation rooted in domestic distributive conflicts. We return to the subject of distributive conflict later and here concern ourselves with the evaluation of collective action as a theoretical framework.

\section{Predictions}

Collective action refers to a strategic setting in which joint cooperation is socially optimal yet difficult to achieve due to competing incentives at the individual level. Thus collective action refers 
to an inevitably broad class of models exhibiting free-riding incentives. One such model, which Aklin and Mildenberger (2020) take as their starting point, is the repeated prisoner's dilemma (PD). Based on their discussion of the repeated PD, Aklin and Mildenberger (2020) make the following empirical predictions: climate cooperation will require the presence of institutions to monitor compliance and coordinate punishment in case of defection (SCA1); states will be willing to undertake climate action only when others behave similarly (SCA2); and states will defect from climate action after observing defection by other states (SCA3).

These predictions center on the role of reciprocity in the repeated PD. Likewise to falsify their predictions the authors bring to bear valuable data on the frequent and widespread adoption of carbon mitigation policies in the absence of mutual cooperation (positive reciprocity). And these policies persist despite high profile defections (negative reciprocity).

The authors' second set of predictions - drawn from what they describe as the weak version of collective action theory - similarly emphasize the role of reciprocity. According to the authors, adherents of this framework adopt a more flexible approach to collective action, acknowledging that it may be one of many forces affecting potential outcomes. We are sympathetic to this position and to the authors' careful engagement with the potential for integrative models of climate policy making. Moreover the evidence brought to bear in this section is both interesting and important for our understanding of climate policy in key decision making contexts.

Nonetheless, as in the case of strong collective action, the authors' second set of predictions emphasize reciprocal behavior as the primary implication of collective action theory. Specifically they predict that policy makers and negotiators will discuss reciprocity in the course of negotiations (WCA1); states will be probabilistically more likely to embrace climate change mitigation when they observe other states doing the same (WCA2, positive reciprocity); and states are more likely to reneg on their carbon mitigation policies after defection of other players, particularly "pivotal" or very influential players such as the United States (WCA3, negative reciprocity).

Below we begin by highlighting three fundamental problems with the authors' reliance on reciprocity as a core feature of collective action. Next we present a more general treatment of 
collective action problems and show that the core feature of these models is underprovision of public goods, rather than any form of reciprocity or even the absence of public goods provision. Rather than falsify models of collective action the empirical regularities in national-level climate policy highlighted in Aklin and Mildenberger (2020) correspond closely to the model's prediction of "some but not enough" climate change mitigation. We conclude with a brief comment on Aklin and Mildenberger (2020)'s interpretation of public opinion data and close with some thoughts on directions for integrating models of collective action and distributive conflict.

We largely agree with many other points raised in Aklin and Mildenberger (2020) and will not discuss those points at great length. For instance, we agree that domestic distributive politics is an interesting source of variation in climate policies and a promising area for future research. We also agree that the literature may be stunted by a single-minded focus on one particular cause of climate inaction. We focus our discussion more narrowly on what collective action models really predict and what would constitute anomalous evidence against them.

\section{Does Collective Action Predict Reciprocity?}

To explain our concerns we briefly re-visit the logic of cooperation in the repeated PD. The repeated PD is a prominent example of how repeated play creates opportunities for cooperation which my otherwise be impossible to sustain. While in the standard PD, there exists no equilibrium in which joint cooperation can be sustained, the same is not true in a repeated setting. By conditioning future cooperation on an opponent's current actions it is possible to support an equilibrium of the repeated prisoner's dilemma characterized by mutual cooperation on the path of play, provided players care sufficiently about future payoffs.

A feature of the repeated PD central to Aklin and Mildenberger (2020)'s analysis 1is described by the influential "Folk Theorem." In broad strokes, the Folk Theorem shows that many outcomes are possible in the repeated PD. In fact, any payoffs that are feasible and weakly better than mutual defection from both players can be sustained as average payoffs in the repeated game if players care enough about the future. The Folk Theorem implies that mutual cooperation can be sustained 
in repeated settings through the use of punishment strategies in which deviation from the proposed cooperative outcome is met with future deviation from some or all other players in the game. In this sense equilibria with cooperation rely on a form of reciprocity: cooperation is met with more cooperation while defection is punished with more defection.

We now describe three key problems which we see in Aklin and Mildenberger (2020)'s focus on the presence or absence of reciprocity. First, doing so confuses potential solutions to the collective action problem with the problem itself. The collective action problem is represented by the PD in which players have individual incentives to defect and mutual cooperation is unsustainable. Equilibria that sustain cooperation using the threat of punishment describe a potential solution to that problem in the form of reciprocal strategies. Seen in this light, an observed lack of reciprocity is no more evidence against the collective action model than the non-existence of a cure for cancer implies the non-existence of cancer.

A second problem with Aklin and Mildenberger's (2020) argument is that they focus on only one possible equilibrium from a very large set of potential equilibria which can be sustained within the repeated PD. In fact one widely-noted challenge in attempting to draw empirical implications from repeated games such as the repeated PD is that these games have many equilibria. The folk theorem not only shows that cooperation is possible, but also that an extraordinarily wide range of outcomes can be sustained in equilibrium provided players are sufficiently patient. In the repeated PD, for instance, there may be many equilibria in which players cooperate along the path of play but it is also an equilibrium for all players to defect in every period, for some players to cooperate on the path of play while others do not, or indeed for certain players to cooperate only in some time periods and defect in others.

The "always defect" equilibrium does not exhibit any kind of reciprocity, so countries are playing this equilibrium we certainly would not observe reciprocity in the data. Though it is difficult to map "cooperate" and "defect" into the complex set of actions available to national policymakers, the current state of affairs is one in which the international community has largely failed to address climate change. Thus, the interpretation that countries are playing the "always 
defect" equilibrium is at least as plausible as an interpretation in which full cooperation has been achieved on the path of play. However, the need to provide richer action sets for policymakers partially motivates the richer model we provide later in this note.

A third problem with the argument is that, even in an equilibrium of the repeated PD exhibiting cooperation, any observed reciprocity is off the equilibrium path of play. Consider the "grim trigger" strategy profile. In this strategy profile, the players cooperate as long as nobody has ever defected and otherwise the players all defect. We must understand strategies in repeated games as complete plans of action which tell us what the players would do given any history, including histories which we should never observe. The grim trigger strategy profile exhibits a form of reciprocity but if we were to observe a group of players actually playing a grim trigger equilibrium then we would never observe any defections. Thus, we would never observe any reciprocity in the actual actions of the players. The main idea of punishment strategies in repeated games is that they are common conjectures about what would happen in the counterfactual scenario in which someone did choose to defect. Thus, even reciprocal equilibria to the repeated PD are consistent with observing unconditional cooperation along the path of play.

To summarize, some solutions to the collective action problem involve reciprocity. Yet the collective action problem itself does not. Furthermore, unconditional defection (in the "always defect" equilibrium) and unconditional cooperation (on the path of play in an equilibrium where cooperation is sustained by the threat of punishment) are equally consistent with Aklin and Mildenberger's (2020)'s chosen benchmark model. Finally reciprocity in a repeated PD applies to actions which occur off the path of play. Deterrence works via the threat rather than the realization of punishment. While it is interesting to note whether there is any evidence of international reciprocity in climate politics, the answer tells us little about whether models of collective action are salient to understanding the challenges of global climate action. 


\section{What Does Collective Action Predict?}

If models of collective action do not predict reciprocity with any certainty, then what empirical patterns would provide support? In this section we describe a core prediction of collective action models, one which is surprisingly consistent with the evidence presented in Aklin and Mildenberger (2020).

In this section we depart from Aklin and Mildenberger (2020)'s focus on the repeated PD as an example of collective action. In fact we agree with Aklin and Mildenberger (2020) that the repeated PD is a limited tool for analyzing the problems of international climate cooperation. In part this stems from the indeterminacy of the Folk Theorem described above. But in part it also stems from simplifying assumptions of the PD which make it difficult to map results from model to empirical world.

In particular, within the prisoner's dilemma 1) there are only two actions, cooperate and defect, and 2) all players are typically assumed to be symmetric.

With respect to the former, consider how one should characterize climate policies that are more than nothing but still insufficient to deal with the problem. Should we call a country that implements such policies a cooperator or defector? The repeated PD does not accurately model the range of actions available to states in the realm of climate. On the latter assumption, player symmetry implies that each participant anticipates identical costs and benefits from climate mitigation. Again this fails to capture key features of climate politics not least of which is the potential for domestic actors or groups to impose differential costs of climate action on policy makers. Adopting a more general conception of public goods then opens the door for a synthetic model of collective action at the international level informed by the sub national distributive conflicts highlighted by Aklin and Mildenberger (2020).

To illustrate this point we describe a more general model of collective action. Consider a set of two or more players (country leaders).

Denote a set of players $N=\{1, \ldots, n\}$ with $n \geq 2$. Each player selects their action, i.e. level of 
carbon emissions, $a_{i} \geq 0$. Preferences are represented by the utility function

$$
u_{i}\left(a_{i}, a_{-i}\right)=\theta_{i} \log \left(a_{i}\right)+\left(1-\theta_{i}\right) \log \left(A-\sum_{j \in N} a_{j}\right)
$$

Here, $a_{-i}$ denotes the actions of all players other than player $i$. Critically, the cost of aggregate carbon emissions accrues to all players regardless of individual players' emission levels. The variable $A>0$ represents the carbon stock or some maximum feasible aggregate emissions level. ${ }^{1}$

The variable $\theta_{i} \in(0,1)$ represents how much player $i$ cares about its own private benefits to carbon consumption relative to conservation. This parameter may reflect both the costs of mitigation - which vary dramatically across settings - as well as the domestic distribution of preferences (distributive conflict). We can think of $\theta_{i}$ the balance of power within each country with respect to environmental or business interests; economic, technological or geological differences between countries; regime differences; or any other factors which lead policy makers to weigh environmental interests differently relative to other interests. ${ }^{2}$

Finally let there be two categories of countries corresponding to two values of $\theta_{i}$. Environmentalist countries have low values of $\theta_{i}$ and industrialist countries have high values, denoted respectively by $\underline{\theta}$ and $\bar{\theta}$ and where $0<\underline{\theta}<\bar{\theta}<1$. Let $m$ denote the number of environmentalist countries with $n-m$ being the number of industrialist countries.

We assume that everything about the game, including the preferences of the players, is common knowledge and characterize the Nash equilibria of the game. In particular we look for equilibria in which consumption depends only on the value of $\theta$ so that $a_{E}$ is the consumption values for all environmentalist countries and $a_{I}$ is the consumption values for all industrialist countries. Full analysis of the model can be found in the appendix. Here we briefly summarize our results.

\section{Proposition 1. In any Nash equilibrium to the game the following are true:}

\footnotetext{
${ }^{1}$ The use of the natural logarithm is only for convenience and the most important thing is simply that the function is concave. This is a standard parameterization used in McAllister and Schnakenberg (2020) and Harrison and Lagunoff (2017) among other papers.

${ }^{2}$ We interpret $\theta_{i}$ in the broadest sense possible in our analysis below. It should be noted however that the results of that analysis do not rely on the presence of distributional conflict. The same results obtain if $\theta_{i}$ is attributed simply to naturally occurring differences in national abatement cost.
} 


\section{The environmentalist countries adopt better climate policies relative to the industrialist} countries

2. If one country becomes more environmentalist then all other countries increase their carbon consumption.

Returning to the empirical evidence described in ??, our proposition establishes that in spite of free-riding incentives, variation in climate policies will persist reflecting the heterogeneity of preferences across countries. The fact that some countries will implement measures to reduce carbon emissions while others do not cannot be interpreted as evidence against free riding. Rather the empirical patterns described in the original paper arise very naturally in collective action models which feature heterogeneity of preferences (a more general preference structure by far than that allowed within the repeated PD for example). The existence or non-existence of free-riding is found by comparing players' actions to the socially optimal actions rather than by comparing players' actions to one another.

A second notable feature of our proposition above is the complete absence of reciprocity. This is seen most clearly in players' best response functions where climate actions behave as strategic substitutes rather than strategic complements. ${ }^{3}$

The fundamental prediction of collective action theories is not that countries do nothing to address collective problems, with or without the possibility of reciprocity. Rather it is that the actions countries do undertake will necessarily fall short of what is socially optimal because individual countries' weighing of costs and benefits fails to account for the effect on the welfare of other countries. This matters since the persistence of global climate change despite significant - albeit insufficient - investments in mitigation is consistent with the predicted outcome of collective action models.

\footnotetext{
${ }^{3}$ Of course, we have presented here a static model. If we repeated the game we could find an equilibrium (for large enough discount factors) where the players took the Pareto optimal actions on the path of play and this would once again exhibit some form of reciprocity off the path of play. The key point is that these considerations are unnecessary in a generally specified model of collective action.
} 


\section{Interpretation of Public Opinion Data}

The public opinion data described in Aklin and Mildenberger (2020) are potentially useful for sorting out what type of equilibrium best describes public perceptions of climate politics. The data show that citizens largely support national efforts at climate change mitigation regardless of other countries' actions. As the survey asks respondents to evaluate counterfactuals in which other countries cooperate or defect it may address our last point above regarding actions off the path of play.

Nonetheless we note two important caveats with respect to Aklin and Mildenberger's (2020) interpretation of the empirical evidence. First, it is not immediately clear what international models of collective action should imply about public opinion. Here is where we agree with the original authors that a model of domestic politics is most useful in understanding variation across citizens. It may be, for instance, that most of the costs of climate action are borne by a powerful minority as the authors suggest.

Second, however, the evidence on whether the public are unconditional cooperators is, at best, mixed. For instance, the authors cite Tingley and Tomz (2014) which indeed shows that the public does not want to defect on climate action in response to others' defections, but also shows that the public supports "extrinsic reciprocity" through linkage of climate change with other issues (c.f. Nordhaus (2015)). In this sense, the evidence from that work is consistent with the idea that the public supports punishment strategies motivated by the need to address problems of collective action. Furthermore, a number of studies show that citizens do value reciprocity in international cooperation and that they are more willing to support cooperative agreements which exhibit this feature (Bechtel, Scheve and van Lieshout, 2019; Bechtel and Scheve, 2013).

\section{Conclusion: Global free riding and distributive conflict}

Aklin and Mildenberger (2020) argue there is limited evidence that global climate policy has been shaped by the logic of collective action. Above we raise three concerns with the empirical impli- 
cations they derive from that model, in particular the emphasis on reciprocity. First, we note that reciprocity is not in fact a feature of collective action models themselves. Rather it is a prediction of a well-known solution to problems of collective action. Second, while reciprocity is a feature of some equilibria of the repeated Prisoners' Dilemma it is just one of many possible strategies which may be observed in the context of collective action. No reciprocity - and defection by all actors - is one equally likely scenario. Finally, we clarify that the reciprocity described in equilibria exhibiting cooperation employ reciprocity off the equilibrium path of play. That is, punishment or defection operates as a latent threat only and should never in fact be observed in these contexts.

Next, we outline our - in agreement with Aklin and Mildenberger (2020) - our concerns regarding the ability of the repeated PD to accurately represent the collective action problem inherent in global climate change policy. We describe a more general, static model of collective action in which costs and benefits may be asymmetric across nations and in which nations can select from a continuous range of policies, i.e. degree of carbon emissions or stringency of mitigation. Characterizing the solution to that model we show how the empirical evidence cited by Aklin and Mildenberger (2020) in their test of the "strong" version of collective action is perfectly reconcilable with - perhaps even supportive of - the logic of collective action.

Finally, while we are generally in agreement with Aklin and Mildenberger (2020)'s approach in their description and analysis of the "weak" version of collective action, we note that the interpretation of the mass public as "unconditional cooperators" relies on a somewhat selective reading of the literature and that, in any case, international models do not make predictions about public opinion. Evidence suggests that the public does support cooperation conditioned on reciprocity, though prefer punishment to occur in non-climate related policy areas.

Aklin and Mildenberger (2020) provide an alternative model where policies are driven by distributive conflict within countries. We entirely agree that this is a good direction for future research. In fact, there are also other plausible theorietical explanations which do not rely on free riding or distributive conflict. For example, short-term electoral incentives may lead policy makers not to internalize the true costs of climate change if their concern for present day political costs outweighs 
the value of (discounted) future benefits.

More generally - as Aklin and Mildenberger (2020) note - models of collective action and distributive conflict are in no way mutually exclusive. As stated in Aklin and Mildenberger (2020): "...collective action and distributive conflict accounts are theoretically compatible within two-level game frameworks, [yet] the relative importance of one or both is an empirical, not theoretical, research task." Here we partially agree. Once the implications of both theories are clear, evaluating their relevance to the application at hand may be a primarily empirical task. But before empirical evidence can be brought to bear we must first ensure that the hypotheses we are testing are deductively valid implications of both theories under consideration. This step of the analysis is where our criticism applies.

With respect to other parts of Aklin and Mildenberger's (2020) argument we find areas of agreement. We conclude by noting some of the ways that models of collective action and distributive conflict may in fact be complementary to one another. One way that distributive conflict models complement collective action models is that they explain the differences between countries that are evident from the model in the previous section. National leaders are weighing various domestic goals against environmental ones and the extent to which they emphasize global environmental cooperation depends on political forces within their country such as the distribution of preferences in the populace, the relative power of various interest groups, the ideological balance of power, and so on. As Aklin and Mildenberger (2020), two-level models provide a useful way to bring all of these considerations into the same model.

Another synthesis of these models is that many distributive conflicts are in fact driven by free riding problems. The reason that many business groups oppose climate action more than the rest of the population is that they believe they would bare a greater share of the costs of those actions while sharing the same benefits as everyone else. In this way, two-level models of climate action are really about similar collective action problems repeated at two different levels. 


\section{References}

Aklin, Michaël and Matto Mildenberger. 2020. "Prisoners of the Wrong Dilemma: Why Distributive Conflict, Not Collective Action, Characterizes the Politics of Climate Change." Global Environmental Politics 20(4):4-27.

URL: https://doi.org/10.1162/glep_a_00578

Bechtel, Michael M and Kenneth F Scheve. 2013. "Mass support for global climate agreements depends on institutional design." Proceedings of the National Academy of Sciences 110(34):13763-13768.

Bechtel, Michael M, Kenneth Scheve and Elisabeth van Lieshout. 2019. "What determines climate policy preferences if reducing greenhouse-gas emissions is a global public Good?" Available at SSRN 3472314 .

Harrison, Rodrigo and Roger Lagunoff. 2017. "Dynamic Mechanism Design for a Global Commons." International Economic Review 58(3):751-782.

URL: https://onlinelibrary.wiley.com/doi/abs/10.1111/iere.12234

McAllister, Jordan H and Keith Schnakenberg. 2020. "Informational and Flexibility in the Design of International Climate Agreements.”.

URL: osf.io/preprints/socarxiv/76mfq

Nordhaus, William. 2015. "Climate Clubs: Overcoming Free-Riding in International Climate Policy.” American Economic Review 105(4):1339-70.

URL: http://www.aeaweb.org/articles?id=10.1257/aer.15000001

Tingley, Dustin and Michael Tomz. 2014. "Conditional Cooperation and Climate Change." Comparative Political Studies 47(3):344-368.

URL: https://doi.org/10.1177/0010414013509571 


\section{Appendix}

Proof of Proposition 1. The best responses of the two types of countries are:

$$
\begin{aligned}
& a_{i}\left(a_{E}, a_{I} \mid \theta_{i}=\underline{\theta}\right)=\underline{\theta}\left(A-(m-1) a_{E}-(n-m) a_{I}\right), \\
& a_{i}\left(a_{E}, a_{I} \mid \theta_{i}=\bar{\theta}\right)=\bar{\theta}\left(A-m a_{E}-(n-m-1) a_{I}\right) .
\end{aligned}
$$

Accounting for the fact that $a_{i}\left(a_{E}, a_{I} \mid \theta_{i}=\underline{\theta}\right)=a_{E}$ and $a_{i}\left(a_{E}, a_{I} \mid \theta_{i}=\bar{\theta}\right)=a_{I}$ gives us the following:

$$
\begin{aligned}
a_{E} & =\frac{\underline{\theta}\left(A-a_{I}(n-m)\right.}{(m-1) \underline{\theta}+1} \\
a_{I} & =\frac{\bar{\theta}\left(A-a_{E} m\right)}{\bar{\theta}(n-m-1)+1} .
\end{aligned}
$$

This gives us a simple system of two equations and two unknowns. Solving the system yields:

$$
\begin{aligned}
a_{E} & =\frac{A \underline{\theta}(1-\bar{\theta})}{\underline{\theta}(m-n \bar{\theta}+\bar{\theta}-1)+\bar{\theta}(n-m-1)+1} \\
a_{I} & =\frac{A(1-\underline{\theta}) \bar{\theta}}{\underline{\theta}(m-n \bar{\theta}+\bar{\theta}-1)+\bar{\theta}(n-m-1)+1}
\end{aligned}
$$

Part 1 of Proposition 1 follows immediately from these expressions. We can see that

$$
\frac{a_{I}}{a_{E}}=\frac{(1-\underline{\theta}) \bar{\theta}}{(1-\bar{\theta}) \underline{\theta}}>1
$$

where the inequality follows from $\bar{\theta}>\underline{\theta}$. Part 2 of Proposition 1 follows from the fact that both actions are increasing in $m$. This follows from the fact that the denominator $\underline{\theta}(m-n \bar{\theta}+\bar{\theta}-1)+$ $\bar{\theta}(n-m-1)+1$ for both expressions in decreasing in $m$. To see this we must verify that:

$$
\underline{\theta}(m-n \bar{\theta}+\bar{\theta}-1)+\bar{\theta}(n-m-1)+1>\underline{\theta}(m+1-n \bar{\theta}+\bar{\theta}-1)+\bar{\theta}(n-m-2)+1
$$


where the right-hand side comes from replacing $m$ with $m+1$ everywhere in the expression. We can easily verify that this holds whenever $\bar{\theta}>\underline{\theta}$. 